\title{
Frequency Weighted Subspace Based System Identification in the Frequency Domain*
}

\author{
Tomas McKelvey \\ Dept. of Electrical Engineering, Linköping University \\ S-581 83 Linköping, Sweden, \\ Phone: +46 13 282461, Fax: +46 13282622 \\ Email: tomas@isy.liu.se.
}

February 27, 1995

\section{Submitted to 34th CDC \\ Report LiTH-ISY-R-1776, ISSN 1400-3902}

\begin{abstract}
Frequency weighting capabilities are introduced in a recent subspace based frequency domain identification algorithm [8]. Weighting matrices constructed from the impulse response of weighting filters are used to weight a Hankel matrix prior of deriving the signal subspace by the singular value decomposition. The resulting algorithm is shown to asymptotically produce models which are frequency weighted balanced. An illustrative example shows the applicability of the method for finite data.
\end{abstract}

Keywords: Identification; Subspace Method; Frequency Weighting; Model Reduction; Singular Value Decomposition.

\section{Introduction}

In most practical identification applications it is important to be able to shape the resulting identification error. Often prior information is available which can be used to improve the model quality. A most common knowledge is the frequency content used in the excitation and the spectral density of the noise sources. For identification in the time domain this is accomplished by prefiltering input and output data [6,12]. For frequency domain methods, i.e when samples of the frequency response functions is the primary data, it is often quite straight forward to use weightings since most methods are best on parametric

\footnotetext{
* This work was supported in part by the Swedish Research Council for Engineering Sciences (TFR), which is gratefully acknowledged.
} 
optimization techniques. The archetypical situation is the minimization of a frequency weighted norm

$$
\hat{G}(z)=\arg \min _{G(z) \in \mathcal{M}} \sum_{k=0}^{M}\left\|F_{k}^{y}\left(G_{k}-G\left(e^{j \omega_{k}}\right)\right) F_{k}^{u}\right\|
$$

where $G_{k}$ are the $M+1$ measured frequency responses and $\hat{G}(z)$ the identified model from some model set $\mathcal{M}$. Here $F_{k}^{y}$ is the output weight matrix and $F_{k}^{u}$ the input weight matrix. For the single input and/or single output case it suffices with one weight matrix since $G(z)$ in this case is vector or scalar valued.

The aim of this contribution is to present an algorithm which produces a suboptimal solution. The algorithm is based on a subspace based identification algorithm [8] augmented with frequency weighting properties. We do this by applying the technique of frequency weighted balanced truncation introduced by Enns [2,3] and further analyzed in [4]. By introducing a particular frequency weighted state space basis followed by state truncation a lower dimensional model results which has a frequency weighted truncation error. Successful applications are reported $[1,15]$ for controller reduction. In $[13,14]$ the frequency weighted balanced realizations are used to interpret which state-space basis estimated models using subspace based algorithms will belong to. Also the direct identification of a lower order model is discussed for various subspace algorithms. Our presentation of Enns's result will be based on [13].

Most model reduction methods assumes the knowledge of a high order system and the task is to find a lower order model with desired model error properties usually given as bounds of the frequency response of the model error. In this contribution we instead assume frequency response measurements of a high order system (possible infinite dimensional) is available and we directly identify a low order model. By using frequency weights we show that the user is free to tune the identification in order to obtain a good fit for important frequency ranges.

The paper has the following outline. In the next section we introduce the problem and fix some notation. Section 3 describes the frequency weighted balanced truncation. In Section 4 we present the identification algorithm and analyze it's properties. Section 5 contains an illustrative example and is followed by the concluding section.

\section{Identification Problem}

Our focus will be on stable discrete time systems. The input-output properties of such a system can represented by the impulse response $g_{k}$ by the convolution

$$
y(t)=\sum_{k=0}^{\infty} g_{k} u(t-k)
$$

where $y(t) \in \mathbb{R}^{p}, u(t) \in \mathbb{R}^{m}$ and $g_{k} \in \mathbb{R}^{p \times m}$. If the system is of finite order $n$ it can be described by a state-space model

$$
\begin{aligned}
x(t+1) & =A x(t)+B u(t) \\
y(t) & =C x(t)+D u(t)
\end{aligned}
$$


where $y(t) \in \mathbb{R}^{p}, u(t) \in \mathbb{R}^{m}$, and $x(t) \in \mathbb{R}^{n}$. The state-space model (2) is a special case of (1) with

$$
g_{k}=\left\{\begin{array}{ll}
D, & k=0 \\
C A^{k-1} B, & k>0
\end{array} .\right.
$$

The frequency response of (1) is

$$
G\left(e^{j \omega}\right)=\sum_{k=0}^{\infty} g_{k} e^{-j \omega k}
$$

which for the state-space model (2) can be written as

$$
G\left(e^{j \omega}\right)=C\left(e^{j \omega} I-A\right)^{-1} B+D .
$$

Our problem formulation is: Given $M+1$, possibly noisy, samples

$$
G_{k}=G\left(e^{j \omega_{k}}\right)+n_{k}, k=0, \ldots, M
$$

of the frequency response of the system at equidistant frequencies $\omega_{k}=\frac{k \pi}{M}, k=$ $0, \ldots, M$, find a finite dimensional state-space system (2) of order $n$, denoted by $\hat{G}$, such that the true system and the identified model are "close". In (6) $G\left(e^{j \omega_{k}}\right)$ represents the system frequency function (4) and $n_{k}$ denotes the noise. Closeness between systems is quantified by the distance between the true and estimated transfer functions and is given by

$$
\left\|F_{y}(z)(\hat{G}-G) F_{u}(z)\right\|_{\infty}
$$

where $F_{u}(z)$ and $F_{y}(z)$ are user supplied stable filters which are used to shape the modeling error.

\section{Frequency Weighted Balanced Model Reduc- tion}

The technique of balanced truncation introduced in [10] and [11] is now a much used method for model reduction. This technique was extended [3, 2] to also enhance the fit in certain frequency regions by user supplied weighting filters. This makes it possible to produce lower order models which are accurate in a frequency region of interest.

Given a model $G(z) \in \mathbb{C}^{p \times m}$ of order $n$, the aim is to find a lower order model $G_{l}(z) \in \mathbb{C}^{p \times m}, l<n$ minimizing the norm

$$
\left\|F_{y}(z)\left(G(z)-G_{l}(z)\right) F_{u}(z)\right\|_{\infty}
$$

where $F_{y}(z) \in \mathbb{C}^{p \times p}$ and $F_{u}(z) \in \mathbb{C}^{m \times m}$ are the output and input frequency filters respectively. These filters are chosen by the user to influence the distribution of the error to certain frequencies. The idea is based on considering the cascaded system

$$
F_{y}(z) G(z) F_{u}(z)
$$

A particular state space basis is determined by diagonalizing parts of the observability and controllability Gramian for the cascaded system (8). Let $(A, B, C, D)$ be a state-space realization of the transfer function $G(z),\left(A_{y}, B_{y}, C_{y}, D_{y}\right)$ a realization for the filter $F_{y}(z)$ and $\left(A_{u}, B_{u}, C_{u}, D_{u}\right)$ the realization corresponding to $F_{u}(z)$. 
Definition 1 The solution $P_{11}$ of the Lyapunov equation:

$$
\begin{aligned}
& \left(\begin{array}{cc}
A & B C_{u} \\
0 & A_{u}
\end{array}\right)\left(\begin{array}{cc}
P_{11} & P_{12} \\
P_{12}^{T} & P_{22}
\end{array}\right)\left(\begin{array}{cc}
A & B C_{u} \\
0 & A_{u}
\end{array}\right)^{T} \\
& +\left(\begin{array}{c}
B D_{u} \\
B_{u}
\end{array}\right)\left(\begin{array}{c}
B D_{u} \\
B_{u}
\end{array}\right)^{T}=\left(\begin{array}{ll}
P_{11} & P_{12} \\
P_{12}^{T} & P_{22}
\end{array}\right)^{T}
\end{aligned}
$$

is the $F_{u}(z)$ weighted controllability Gramian denoted by

$$
W_{c}^{u}=P_{11} \text {. }
$$

Of course we also have the dual Gramian.

Definition 2 The solution $Q_{11}$ of the Lyapunov equation:

$$
\begin{gathered}
\left(\begin{array}{cc}
A & 0 \\
B_{y} C & A_{y}
\end{array}\right)^{T}\left(\begin{array}{ll}
Q_{11} & Q_{12} \\
Q_{12}^{T} & Q_{22}
\end{array}\right)\left(\begin{array}{cc}
A & 0 \\
B_{y} C & A_{y}
\end{array}\right) \\
+\left(\begin{array}{cc}
D_{y} C & C_{y}
\end{array}\right)^{T}\left(\begin{array}{ll}
D_{y} C & C_{y}
\end{array}\right)=\left(\begin{array}{ll}
Q_{11} & Q_{12} \\
Q_{12}^{T} & Q_{22}
\end{array}\right)
\end{gathered}
$$

is the $F_{y}(z)$ weighted observability Gramian denoted by

$$
W_{o}^{y}=Q_{11}
$$

If the realization of $G(z)$ undergoes a similarity transformation $T \in \mathbb{R}^{n \times n}$

$$
\begin{aligned}
& A \rightarrow T A T^{-1} \\
& B \rightarrow T B \\
& C \rightarrow C T^{-1}
\end{aligned}
$$

it is easy to show that

$$
\begin{aligned}
& W_{c}^{u} \rightarrow T^{-1} W_{c}^{u} T^{-T} \\
& W_{o}^{y} \rightarrow T^{T} W_{o}^{y} T .
\end{aligned}
$$

In a similar fashion as for the classical balancing problem [10] it is always possible to make the two Gramian matrices diagonal and equal for a proper choice of transformation matrix $T$.

Definition 3 A system $G(z)$ with realization $(A, B, C, D)$ is frequency weighted balanced if

$$
W_{c}^{u}=W_{o}^{y}=\Sigma\left(F_{u}, F_{y}\right)
$$

where $\Sigma\left(F_{u}, F_{y}\right)=\operatorname{diag}\left(\sigma_{1}, \sigma_{2}, \ldots, \sigma_{n}\right)$ are the frequency weighted Hankel singular values in a non-increasing order denoted by

$$
\sigma_{k}\left(F_{u}, F_{y}\right)
$$

From (9) we notice that the eigenvalues of $W_{c}^{u} W_{o}^{y}$ always equal the squared frequency weighted Hankel singular values. It is also straight forward to see that $\Sigma\left(F_{u}, F_{y}\right)$ also is independent of the realization of $G(z)$ or the realizations of the filters $F_{y}(z)$ and $F_{u}(z)$. 
Let us now consider a state-truncation of a realization given in a frequency balanced realization. This leads to a reduced order model. If we partition the state-space matrices

$$
\begin{aligned}
& A=\left(\begin{array}{ll}
A_{11} & A_{12} \\
A_{21} & A_{22}
\end{array}\right), \quad B=\left(\begin{array}{l}
B_{1} \\
B_{2}
\end{array}\right) \\
& C=\left(\begin{array}{ll}
C_{1} & C_{2}
\end{array}\right),
\end{aligned}
$$

with $A_{11} \in \mathbb{R}^{l \times l}, B_{1} \in \mathbb{R}^{l \times m}$ and $C_{1} \in \mathbb{R}^{p \times l}$, a reduced order model is given by the realization $\left(A_{11}, B_{1}, C_{1}, D\right)$. The reduced order transfer function is then

$$
G_{l}(z)=C_{1}\left(z I-A_{11}\right)^{-1} B_{1}+D .
$$

A recent result gives a bound on the truncation error.

Theorem 1 ([4]) Let $G(z)$ be a stable transfer function of order $n$ and let $F_{u}(z)$ and $F_{y}(z)$ be stable weighting functions. Also let $G_{l}(z)$ be the frequency weighted balanced truncation (10) of order $l$. Assume $G_{l}(z)$ is stable. Then the following error bound holds:

$$
\left\|F_{y}(z)\left(G(z)-G_{l}(z)\right) F_{u}(z)\right\|_{\infty} \leq 2 \sum_{k=l+1}^{n} \sqrt{\sigma_{k}^{2}+\left(\alpha_{k}+\beta_{k}\right) \sigma_{k}^{3 / 2}+\alpha_{k} \beta_{k} \sigma_{k}}
$$

where $\sigma_{k}=\sigma_{k}\left(F_{y}, F_{u}\right)$, and $\alpha_{k}$ and $\beta_{k}$ are finite.

For brevity we have omitted the expressions for $\alpha_{k}$ and $\beta_{k}$. The full expressions can be found in [4].

\section{Frequency Weighted Identification}

In this subsection we will introduce a frequency weighted variation of the subspace based algorithm [7]. This algorithm is based on the geometrical properties of a Hankel matrix $\hat{H}_{q r}$ constructed by the coefficients resulting from the inverse discrete Fourier transform of the noise-free samples of the true system frequency function. It is straight forward [8] to show that

$$
\hat{H}_{q r}=\hat{\mathcal{O}}_{q}\left(I-\hat{A}^{2 M}\right)^{-1} \hat{\mathcal{C}}_{r}
$$

where

$$
\hat{\mathcal{O}}_{q}=\left(\begin{array}{c}
\hat{C} \\
\hat{C} \hat{A} \\
\vdots \\
\hat{C} \hat{A}^{q-1}
\end{array}\right)
$$

is the $q$-block extended observability matrix and

$$
\hat{\mathcal{C}}_{r}=\left(\begin{array}{llll}
\hat{B} & \hat{A} \hat{B} & \ldots & \hat{A}^{r-1} \hat{B}
\end{array}\right)
$$

is the $r$-block column controllability matrix. This shows that $\hat{H}_{q r}$ has rank $n$, the system order. The column space of $H_{q r}$ thus equals the column space of $\hat{\mathcal{O}}_{q}$. By using the singular value decomposition

$$
\hat{H}_{q r}=\hat{U}_{s} \hat{\Sigma}_{s} \hat{V}_{s}^{T}
$$


where $U_{s} \in \mathbb{R}^{q p \times n}, U_{s} \Sigma_{s}^{1 / 2}$ is the extended observability matrix of some realization of the true system. By utilizing the block shift properties of this matrix the $\hat{A}$ and $\hat{C}$ matrix can be determined [5]. $\hat{B}$ and $\hat{D}$ matrices can then be recovered either by solving a linear least-squares problem or by utilizing the geometrical properties of the right factor of the SVD.

This algorithm will now be augmented with frequency weighting capabilities. Let us first introduce two lower triangular block-Toeplitz matrices with elements from the impulse response of the two stable frequency filters.

$$
\begin{gathered}
F_{y}=\left(\begin{array}{ccccc}
D_{y} & 0 & 0 & \ldots & 0 \\
C_{y} B_{y} & D_{y} & 0 & \ldots & 0 \\
C_{y} A_{y} B_{y} & C_{y} B_{y} & D_{y} & \ldots & 0 \\
\ldots & \cdots & \ldots & \ldots & \ldots \\
C_{y} A_{y}^{q-2} B_{y} & C_{y} A_{y}^{q-3} B_{y} & C_{y} A_{y}^{q-4} B_{y} & \ldots & D_{y}
\end{array}\right) \in \mathbb{R}^{q p \times q p} \\
F_{u}=\left(\begin{array}{ccccc}
D_{u} & 0 & 0 & \ldots & 0 \\
C_{u} B_{u} & D_{u} & 0 & \ldots & 0 \\
C_{u} A_{u} B_{u} & C_{u} B_{u} & D_{u} & \ldots & 0 \\
\ldots & \ldots & \ldots & \ldots & \ldots \\
C_{u} A_{u}^{r-2} B_{u} & C_{u} A_{u}^{r-3} B_{u} & C_{u} A_{u}^{r-4} B_{u} & \ldots & D_{u}
\end{array}\right) \in \mathbb{R}^{r m \times r m}
\end{gathered}
$$

Using $F_{y}$ and $F_{u}$ we can formulate a frequency weighted identification algorithm. Assume that frequency response data $G_{k}$ on a set of uniformly spaced frequencies, $\omega_{k}=\frac{\pi k}{M}, k=0, \ldots, M$ are given. Since $G$ is a transfer function with a real valued impulse response (1), frequency response data on $[0, \pi]$ can be extended to $[0,2 \pi]$ which forms the first step of the algorithm.

\section{Algorithm 1}

1.

$$
G_{M+k}:=G_{M-k}^{*}, \quad k=1, \ldots, M-1
$$

where $(\cdot)^{*}$ denotes complex conjugate.

2. Let $\hat{h}_{i}$ be defined by the $2 M$-point Inverse Discrete Fourier Transform (IDFT)

$$
\hat{h}_{i}=\frac{1}{2 M} \sum_{k=0}^{2 M-1} G_{k} e^{j 2 \pi i k / 2 M}, i=0, \ldots, q+r-1 .
$$

3. Let the block Hankel matrix $\hat{H}_{q r}$ be defined as

$$
\hat{H}_{q r}=\left(\begin{array}{cccc}
\hat{h}_{1} & \hat{h}_{2} & \ldots & \hat{h}_{r} \\
\hat{h}_{2} & \hat{h}_{3} & \ldots & \hat{h}_{r+1} \\
\vdots & \vdots & \ddots & \vdots \\
\hat{h}_{q} & \hat{h}_{q+1} & \ldots & \hat{h}_{q+r-1}
\end{array}\right) \in \mathbb{R}^{q p \times r m}
$$

with number of block rows $q>n$ and block columns $r \geq n$. The size of $\hat{H}_{q r}$ is limited by $q+r \leq M$. 
4. Let the singular value decomposition of the weighted Hankel matrix $F_{y} \hat{H}_{q r} F_{u}$ be denoted by

$$
F_{y} \hat{H}_{q r} F_{u}=\left[\begin{array}{ll}
\hat{U}_{s} & \hat{U}_{o}
\end{array}\right]\left(\begin{array}{cc}
\hat{\Sigma}_{s} & 0 \\
0 & \hat{\Sigma}_{o}
\end{array}\right)\left(\begin{array}{c}
\hat{V}_{s}^{T} \\
\hat{V}_{o}^{T}
\end{array}\right)
$$

where $\hat{\Sigma}_{s}$ contains the $n$ largest singular values.

5.

$$
\begin{aligned}
\hat{A} & =\left(J_{1} F_{y}^{-1} \hat{U}_{s} \hat{\Sigma}_{s}^{1 / 2}\right)^{\dagger} J_{2} F_{y}^{-1} \hat{U}_{s} \hat{\Sigma}_{s}^{1 / 2} \\
\hat{C} & =J_{3} F_{y}^{-1} \hat{U}_{s} \Sigma_{s}^{1 / 2}
\end{aligned}
$$

6 .

$$
\begin{aligned}
& \hat{B}=\left(I-\hat{A}^{2 M}\right) \hat{\Sigma}_{s}^{1 / 2} \hat{V}_{s}^{T} F_{u}^{-1} J_{4} \\
& \hat{D}=\hat{h}_{0}-\hat{C} \hat{A}^{2 M-1}\left(I-\hat{A}^{2 M}\right)^{-1} \hat{B}
\end{aligned}
$$

where

$$
\begin{aligned}
& J_{1}=\left(\begin{array}{ll}
I_{(q-1) p} & 0_{(q-1) p \times p}
\end{array}\right), \quad J_{2}=\left(\begin{array}{cc}
0_{(q-1) p \times p} & I_{(q-1) p}
\end{array}\right) \\
& J_{3}=\left(\begin{array}{ll}
I_{p} & 0_{p \times(q-1) p}
\end{array}\right), \quad J_{4}=\left(\begin{array}{c}
I_{m} \\
0_{(r-1) m \times m}
\end{array}\right)
\end{aligned}
$$

and $I_{i}$ denotes the $i \times i$ identity matrix, $0_{i \times j}$ denotes the $i \times j$ zero matrix and $X^{\dagger}=\left(X^{T} X\right)^{-1} X^{T}$ denotes the Moore-Penrose pseudo-inverse of the full rank matrix $X$.

We also can consider an alternative way of determining $B$ and $D$ by using a frequency weighted least-squares solution.

\section{Algorithm 2}

1. Steps 1-5 in Algorithm 1.

2.

$$
\hat{B}, \hat{D}=\arg \min _{B, D} \sum_{k=0}^{M}\left\|F_{y}\left(e^{j \omega_{k}}\right)\left(G_{k}-D-\hat{C}\left(e^{j \omega_{k}} I-\hat{A}\right)^{-1} B\right) F_{u}\left(e^{j \omega_{k}}\right)\right\|_{F}^{2}
$$

Denote by $\hat{G}$ the identified transfer function

$$
\hat{G}(z)=\hat{C}(z I-\hat{A})^{-1} \hat{B}+\hat{D} .
$$

When applied to data which is noise free and from a system of order $n$ these two algorithms will deliver state-space realizations which are frequency weighted balanced as given in Definition 3 when $q, r \rightarrow \infty$. Before deriving this result we look at an alternative derivation of the frequency weighted Gramian matrices.

As well as the the standard Gramian matrices can be expressed as infinite sums, the frequency weighted Gramians can also be described as an infinite sum $[14,13]$. 
Lemma $1([\mathbf{1 3}])$ If $G(z), F_{u}(z)$ and $F_{y}(z)$ are stable then

$$
\begin{gathered}
W_{o}^{y}=\lim _{q \rightarrow \infty} \mathcal{O}_{q}^{T} F_{y}^{T} F_{y} \mathcal{O}_{q} \\
W_{c}^{u}=\lim _{r \rightarrow \infty} \mathcal{C}_{r} F_{u} F_{u}^{T} \mathcal{C}_{r}^{T}
\end{gathered}
$$

where $\mathcal{O}_{q}$ and $\mathcal{C}_{r}$ are the extended observability and controllability matrices of the realization $(A, B, C, D)$ of $G(z)$.

We are now ready to present the main result of this paper.

Theorem 2 Suppose $M+1$ equidistant frequency samples of a stable linear system $G$ of order $n$ are given. Let $F_{u}(z)$ and $F_{y}(z)$ be any stable filters with $D_{u}$ and $D_{y}$ matrices of full rank. Then

(i) If $q>n, r \geq n$ and $M \geq q+r$, the estimated system $\hat{G}$ using Algorithms 1 or 2 will equal $G$ if the frequency response $G_{k}$ is noise-free.

(ii) The realization of $\hat{G}$ will be frequency weighted balanced and $\hat{\Sigma}_{s} \rightarrow \Sigma\left(F_{u}, F_{y}\right)$ as $M, q, r \rightarrow \infty$.

Proof: $\hat{H}_{q r}$ has rank $n$ [8]. Since $D_{u}$ and $D_{y}$ are of full rank $F_{u}$ and $F_{u}$ are matrices of full rank independently of $q$ and $r$. Hence $F_{y} \hat{H}_{q r} F_{u}$ is still a matrix of rank $n$. Using (16) we can write

$$
F_{y} \hat{H}_{q r} F_{u}=\hat{U}_{s} \hat{\Sigma}_{s} \hat{V}_{s}^{T}
$$

In (17) and (18) we implicitly use

$$
\hat{\mathcal{O}}_{q}=F_{y}^{-1} \hat{U}_{s} \hat{\Sigma}_{s}^{1 / 2}
$$

as the extended observability matrix. (19) also implicitly defines

$$
\hat{\mathcal{C}}_{r}=\left(I-\hat{A}^{2 M}\right) \hat{\Sigma}_{s}^{1 / 2} \hat{V}_{s}^{T} F_{u}^{-1}
$$

as the controllability matrix. Hence we obtain

$$
\hat{\mathcal{O}}_{q}\left(I-\hat{A}^{2 M}\right)^{-1} \hat{\mathcal{C}}_{r}=\hat{H}_{q r} .
$$

(i) now follows from Theorem 3.1 in [8].

For the second part (ii) let $M, q, r \rightarrow \infty$ and drop the indices $q$ and $r$. Then $A^{2 M} \rightarrow 0$ and consequently using (24) and (25) we can write

$$
\mathcal{C} F_{u}=\hat{\Sigma}_{s}^{1 / 2} \hat{V}_{s}^{T}
$$

and

$$
F_{y} \mathcal{O}=\hat{U}_{s} \hat{\Sigma}_{s}^{1 / 2} .
$$

Finally using Lemma 1 we obtain the frequency weighted Gramians as

$$
W_{o}^{y}=\mathcal{O}^{T} F_{y}^{T} F_{y} \mathcal{O}=\hat{\Sigma}_{s}
$$

and

$$
W_{c}^{u}=\mathcal{C} F_{u} F_{u}^{T} \mathcal{C}^{T}=\hat{\Sigma}_{s}
$$


since $\hat{U}_{s}^{T} \hat{U}_{s}=I$ and $\hat{V}_{s}^{T} \hat{V}_{s}=I$. Both Gramians are diagonal and equal which imply that the identified state-space model is frequency weighted balanced and that

$$
\hat{\Sigma}_{s}=\Sigma\left(F_{u}, F_{y}\right) .
$$

In Algorithm $2 A$ and $C$ are determined as in Algorithm 1. Since the data is assumed noise free $\hat{B}$ and $\hat{D}$ are the unique matrices which makes the sum (23) identical zero the same results directly follows.

Notice that the frequency filters $F_{u}(z)$ and $F_{y}(z)$ only influence the realization obtained and not the transfer function itself. This holds only when dealing with noise free data and if the system has order $n$. In practice these two conditions always are violated and now the frequency filters will play an important role. In this case the singular values of $\hat{\Sigma}_{o}$ will not be zero and the algorithm will produce a model which is close to the truncated frequency weighted balanced realization.

Remark 1 By applying the stochastic analysis given in $[8,9]$ it follows that the stated result also holds with probability 1 if the measurements $G_{k}$ are corrupted with zero mean random noise with bounded second moments and we let $q, r=$ $O\left(M^{1 / 3}(\log M)^{-\beta}\right)$ for some $\beta>1 / 3$.

If applied to data from an infinite dimensional system the unweighted identification algorithm delivers models which converge to the truncated balanced realization [9]. It would thus be appealing to extend this result for the new frequency weighted algorithm and show that the frequency weighted algorithm asymptotically will deliver models which are the frequency weighted balanced truncation of an infinite dimensional system.

\section{$5 \quad$ Applicability to Finite Data}

In the previous section we have demonstrated that Algorithms 1 and 2 have the desired asymptotic behavior; frequency weighted balanced realizations will result from the identification. In this section we will investigate what properties the algorithm possess when used on finite data. We do this by considering an example.

Let $G$ be a fourth order model with a frequency response with two resonant peaks. The following state-space model represents $G$

$$
\begin{aligned}
x(t+1) & =\left(\begin{array}{cccc}
0.8876 & 0.4494 & 0 & 0 \\
-0.4494 & 0.7978 & 0 & 0 \\
0 & 0 & -0.6129 & 0.0645 \\
0 & 0 & -6.4516 & -0.7419
\end{array}\right) x(t)+\left(\begin{array}{l}
0.2247 \\
0.8989 \\
0.0323 \\
0.1290
\end{array}\right) u(t) \\
y(t) & =\left(\begin{array}{ccccc}
0.4719 & 0.1124 & 9.6774 & 1.6129
\end{array}\right) x(t)+0.9626 u(t) .
\end{aligned}
$$

The transfer function of $G$ is sampled at 101 equidistant frequency points including frequency 0 and $\pi$. The solid line in Figure 1 represents the magnitude of the frequency function. Let us consider four different identification cases:

Case 1 Algorithm 1 with low pass frequency filter.

Case 2 Algorithm 1 with high pass frequency filter. 
Case 3 Algorithm 2 with low pass frequency filter.

Case 4 Algorithm 2 with high pass frequency filter.

Since we are dealing with a SISO system we let $F_{u}(z)=F_{y}(z)$ and use a fifth order standard Butterworth filter with cut-off frequency $0.4 / \pi \mathrm{rad} / \mathrm{s}$ in the lowpass form for Case 1 and 3 and in high-pass form for the other two cases. In Figure 1 the results of the four cases are presented. The solid graph is the magnitude of the original fourth order system. The dashed graph represents the estimated second order model for the four different cases. The magnitude of the frequency filters are shown as dotted line and the dash-dotted line is the magnitude of the difference between the original model and the second order estimated model. In all four cases the peak is correctly estimated which indicates that the pole locations in the desired frequency band have been properly found. The final error in the weighted frequency band is lower for case 3 and 4 . This stems from improved DC-level and zero locations by the additional weighted least-squares step found in Algorithm 2. Based on this example it is clear that the frequency weights $F_{y}$ and $F_{u}$ indeed can make the algorithms focus on a selected frequency range.

\section{Conclusions and Open Problems}

In this paper we have shown how the identification algorithm [8] can be augmented with frequency weighting capabilities. We do this by using a technique closely related to frequency weighted balanced model reduction [2]. We show that as the size of the Hankel matrix tends to infinity the identification method yields models which are frequency weighted balanced. By considering an example, the applicability of the new frequency weighted algorithms to the case of finite data has been shown to be adequate. The algorithms are thus well suited for lower order modeling directly from frequency data originating from systems of high complexity. This approach is thus a viable alternative to the two step approach of higher order modeling followed by a model reduction step. It is still an open question if the algorithm, when applied to data from infinite dimensional systems, will yield finite dimensional models which are frequency weighted balanced truncation of the original system.

\section{References}

[1] B. D. O. Anderson and J. B. Moore. Optimal Control - Linear Quadratic Methods. Prentice-Hall, Englewood Cliffs, New Jersey, 1989.

[2] D. F. Enns. Model reduction for control system design. PhD thesis, Department of Aeronautics and Astronautics, Stanford University, Stanford, CA, 1984.

[3] D. F. Enns. Model reduction with balanced realizations: An error bound and a frequency weighted generalization. In Proc. of the 23rd IEEE Conference on Decision and Control, Las Vegas, NV, pages 127-132, 1984. 

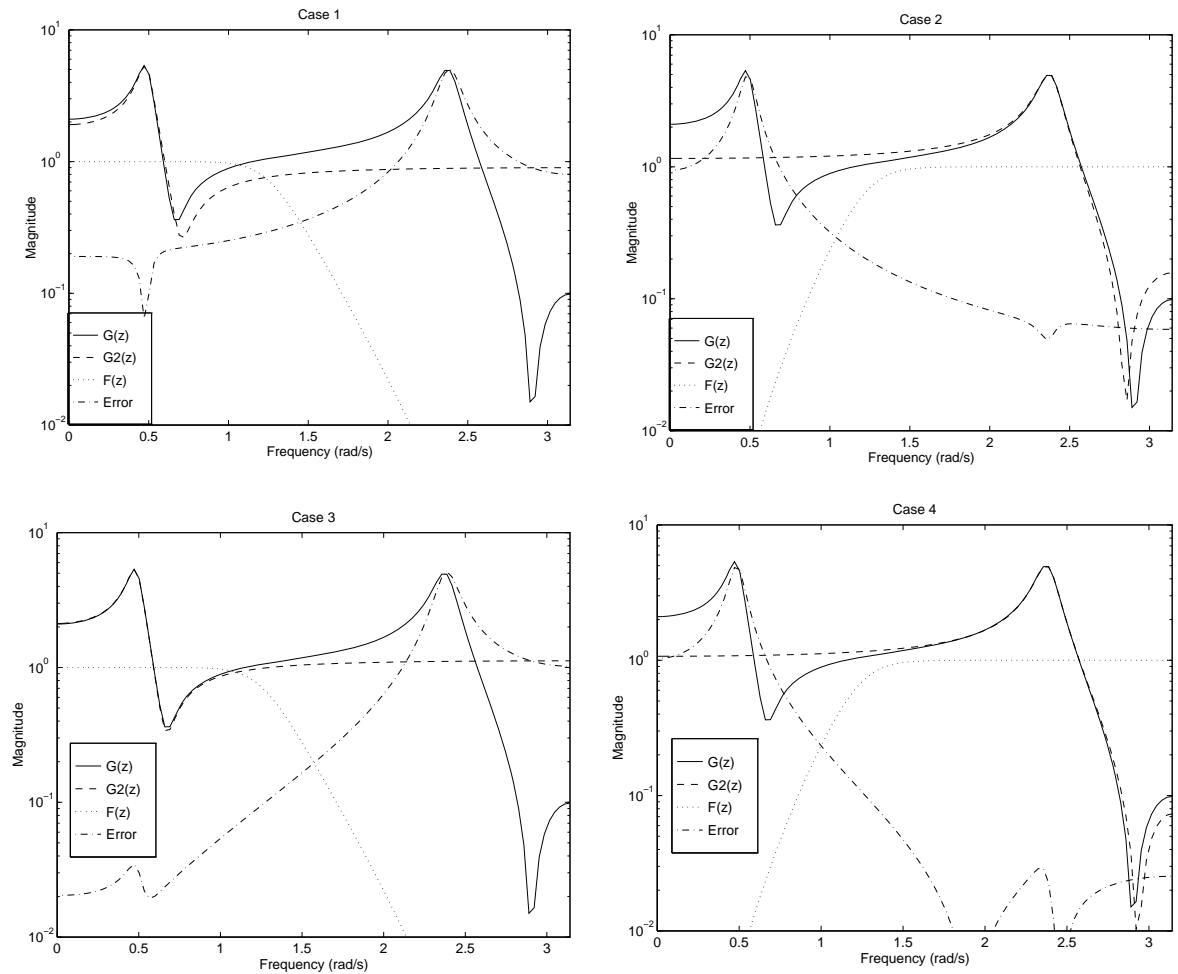

Figure 1: Results of the four different identification cases: Upper left; case 1 using Alg. 1 with low-pass weighting. Upper right case 2; Alg. 1 with highpass weighting. Lower left case 3 ; Alg. 2 with low-pass weighting. Lower right case 4; Alg. 2 with high-pass weighting. The solid graphs are the magnitude of the original fourth order system. The dashed graphs represents the estimated second order models. The magnitude of the frequency filters are shown as dotted lines and the dash-dotted lines are the magnitude of the difference between the original model and the second order estimated model.

[4] S. W. Kim, B. D. O. Anderson, and A. G. Madievski. Error bound for transfer function order reduction using frequency weighted balanced truncation. Systems \& Control Letters, 24(3):183-192, February 1995.

[5] S. Y. Kung. A new identification and model reduction algorithm via singular value decomposition. In Proc. of 12th Asilomar Conference on Circuits, Systems and Computers, Pacific Grove, CA, pages 705-714, 1978.

[6] L. Ljung. System Identification: Theory for the User. Prentice-Hall, Englewood Cliffs, New Jersey, 1987.

[7] T. McKelvey and H. Akçay. An efficient frequency domain state-space identification algorithm. In Proc. 33rd IEEE Conference on Decision and Control, Lake Buena Vista, Florida, pages 3359-3364, December 1994. 
[8] T. McKelvey and H. Akçay. An efficient frequency domain state-space identification algorithm: Robustness and stochastic analysis. In Proc. 33rd IEEE Conference on Decision and Control, Lake Buena Vista, Florida, pages 3348-3353, December 1994.

[9] T. McKelvey, H. Akçay, and L. Ljung. Identification of infinite dimensional systems from frequency response data. Technical report, Report LiTH-ISYR-1681, Dep. of EE, Linköping University, S-581 83 Linköping, Sweden, 1994. Submitted to Automatica.

[10] B. C. Moore. Principal component analysis in linear systems: Controllability, observability, and model reduction. IEEE Trans. on Automatic Control, 26(1):17-32, 1981.

[11] L. Pernebo and L. M. Silverman. Model reduction via balanced state space representations. IEEE Trans. on Automatic Control, 27(2):382-387, 1982.

[12] T. Söderström and P. Stoica. System Identification. Prentice-Hall International, Hemel Hempstead, Hertfordshire, 1989.

[13] P. Van Overschee. Subspace Identification, Theory - Implementation - Application. PhD thesis, Katholieke Universiteit Leuven, Kard. Mercierlaan 94, 3030 Leuven (Heverlee), Belgium, February 1995.

[14] P. Van Overschee and B. De Moor. A unifying theorem for subspace identification algorithms and its interpretation. In Proceedings of the 10th IFAC Symposium on System Identification, volume 2, pages 145-150, Copenhagen, Denmark, July 1994.

[15] P. M. R. Wortelboer and O. H. Bosgra. Generalized frequency weighted balanced reduction. In O. H. Bosgra and P. M. J. Van den Hof, editors, Selected Topics in Identification, Modeling and Control, volume 5, pages 29-36. Delft University Press, 1992. 